\title{
A novel biosimulator based on ex vivo porcine lungs for training in peripheral tissue sampling using endobronchial ultrasonography with a guide sheath
}

\author{
Tsukasa Ishiwata ${ }^{1}$, Takahiro Nakajima ${ }^{2}$, Jiro Terada $^{1}$, Koichiro Tatsumi ${ }^{1}$ \\ ${ }^{1}$ Department of Respirology, ${ }^{2}$ Department of General Thoracic Surgery, Graduate School of Medicine, Chiba University, Chiba, Japan \\ Contributions: (I) Conception and design: T Ishiwata, T Nakajima; (II) Administrative support: J Terada, K Tatsumi; (III) Provision of study materials \\ or patients: T Ishiwata, T Nakajima, J Terada; (IV) Collection and assembly of data: T Ishiwata; (V) Data analysis and interpretation: T Ishiwata; (VI) \\ Manuscript writing: All authors; (VII) Final approval of manuscript: All authors. \\ Correspondence to: Tsukasa Ishiwata, MD, PhD. Department of Respirology, Graduate School of Medicine, Chiba University, 1-8-1 Inohana, Chuo- \\ ku, Chiba 260-8670, Japan. Email: Tsukasa.ishiwata@uhnresearch.ca.
}

Background: Although radial probe endobronchial ultrasonography (EBUS) with a guide sheath (GS; EBUS-GS) is widely used for sampling peripheral pulmonary lesions (PPLs), a standard training model for EBUS-GS remains to be developed. The purpose of this study was to evaluate the feasibility of a novel pulmonary biosimulator for hands-on training in peripheral tissue sampling using EBUS-GS

Methods: We established a novel biosimulator for EBUS-GS using porcine lungs. The simulator was equipped with multiple pseudo PPLs that were created using blue agar solution injected through GS inserted in a bronchoscope. A total of 12 voluntary trainees participated in a hands-on training course using the biosimulator. The size of samples acquired using biopsy forceps were compared between initial and posttraining biopsies, and trainee satisfaction with the biosimulator and training program were evaluated using a questionnaire.

Results: Under the guidance of a trainer, all trainees successfully detected pseudo PPLs using radial probe EBUS before the initial biopsy, and 11 trainees acquired samples from the target lesions during the initial biopsy. Post-training biopsy samples were larger than the initial samples for eight trainees. The results of the questionnaire revealed that all trainees were satisfied with the biosimulator. Moreover, eight trainees who had previously participated in another hands-on EBUS-GS training program involving a synthetic phantom model showed greater satisfaction for the biosimulator.

Conclusions: A hands-on training program using the novel biosimulator assessed in this study could aid clinicians in improving their skills for EBUS-GS and acquiring larger peripheral tissue samples using biopsy forceps inserted through GS.

Keywords: Bronchoscopy; biosimulator; endobronchial ultrasonography (EBUS); guide sheath (GS); peripheral pulmonary lesions (PPLs)

Submitted May 07, 2019. Accepted for publication Sep 24, 2019.

doi: $10.21037 /$ jtd.2019.10.05

View this article at: http://dx.doi.org/10.21037/jtd.2019.10.05

\section{Introduction}

A sampling method using radial probe endobronchial ultrasonography (EBUS) has been established as a promising bronchoscopic approach for the diagnosis of peripheral pulmonary lesions (PPLs) (1). The combined use of a guide sheath (GS) and radial probe EBUS (EBUSGS), which provides a $360^{\circ}$ sonographic image of the surrounding lung tissue, is an effective modality that allows bronchoscopists to perform repeated tissue sampling from the same position. With the development of EBUS-GS, 


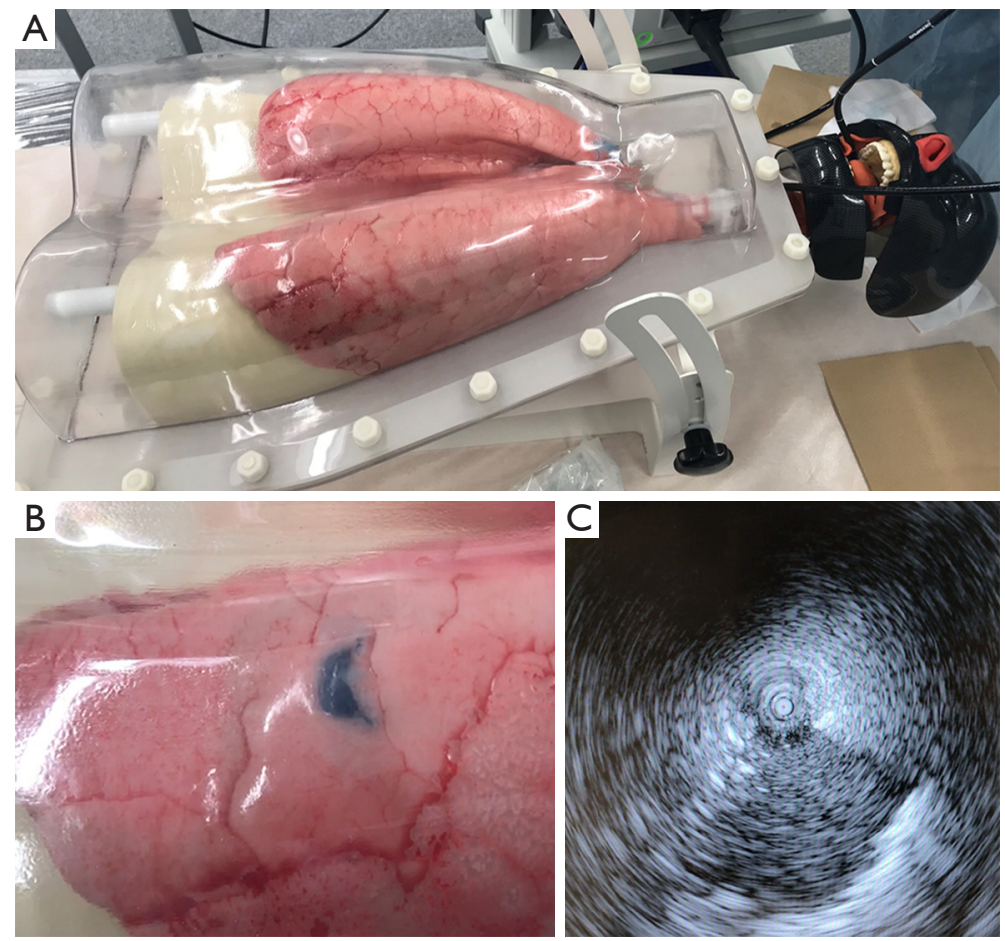

Figure 1 A novel biosimulator with pseudo PPLs created for hands-on training in peripheral tissue sampling using EBUS-GS. (A) The ArtiCHEST $^{\mathrm{TM}}$ system is used for fixing and inflating porcine lungs; (B) blue agar solution is injected through GS inserted in a bronchoscope. The locations of PPLs can be observed from the lung surface; (C) clear images of PPLs are obtained by a radial EBUS probe. PPLs, peripheral pulmonary lesions; GS, guide sheath; EBUS-GS, endobronchial ultrasonography with a guide sheath.

the diagnostic yield for PPLs has greatly improved and is currently better than that achieved with conventional bronchoscopy (2). However, novel techniques for training beginners in the use of new devices are necessary.

Several guidelines concerning diagnostic bronchoscopy recommend that each learner should undergo brief but intense training, including hands-on training (3-6). Accordingly, EBUS-GS should be also performed with a fair amount of dexterity, because GS can often break if clinicians have inadequate knowledge about the device (7). Furthermore, the diagnostic yield of EBUS-GS can improve with an increase in the number of procedures performed, particularly among beginners (7). In summary, training is considered one of the most important factors contributing to an improved diagnostic yield of EBUS-GS for PPLs (8). However, the most suitable model for providing hands-on training in EBUS-GS remains to be elucidated.

In the present study, we established a novel biosimulator based on ex vivo porcine lungs with pseudo PPLs. We hypothesized hands-on training using the new model contribute to bronchoscopists acquiring the knowledges and techniques in EBUS-GS for PPLs.

\section{Methods}

We established a training model for EBUS-GS procedures. The feasibility of the training model was prospectively evaluated in a hands-on training program in June, 2018.

\section{Training model (Figures 1,2)}

The ArtiCHEST ${ }^{\mathrm{TM}}$ lung phantom system (HARADA Corporation, Japan) was used for preparing the biosimulator. This system consists of a box in which porcine trachea and lung lobes with an artificial diaphragm can be set, as well as an aspiration pump that creates a negative pressure for lung inflation. The porcine organ was purchased from a supplier providing animal organs for research (Tokyo Shibaura Zouki Corporation, Japan).

After setting up the training simulator, we created pseudo PPLs in the porcine lungs using the following method (Figure 2). First, GS was placed in a peripheral 


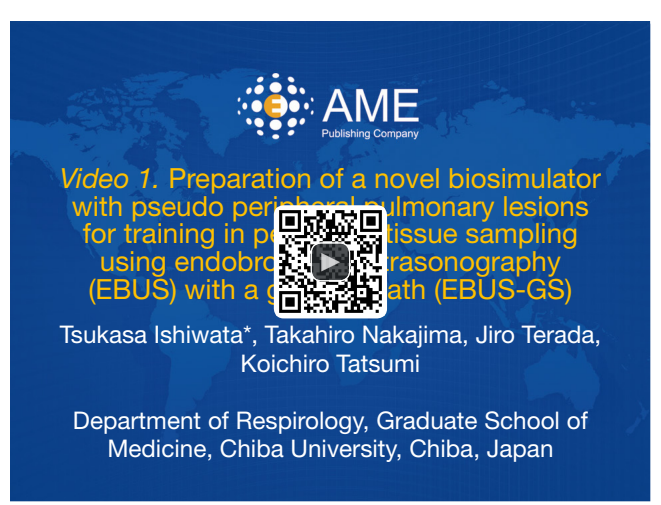

Figure 2 Preparation of a novel biosimulator with pseudo peripheral pulmonary lesions for training in peripheral tissue sampling using endobronchial ultrasonography (EBUS) with a guide sheath (EBUS-GS) (9).

Available online: http://www.asvide.com/watch/32997

region through bronchoscopy, and it was moved around to enable confirmation of the position of its tip from outside the lungs. Then, $10 \mathrm{~mL}$ of $2 \%$ agar solution mixed with indigocarmine (blue color) was prepared using a hot stirrer and injected through GS. Any excess endobronchial agar solution observed from the bronchial lumen was removed, and the solution was cooled for $30 \mathrm{~min}$ at room temperature for solidification. A preliminary study revealed that the optimal concentration of the agar solution for creating pseudo PPLs in the porcine lungs was $2 \%$. If a concentration of $<1.5 \%$ was used, some solution returned to the main bronchus before the pseudo PPLs could be created in the lung lobes. On the other hand, if a concentration of $>3 \%$ was used, the pseudo PPLs were crumbly and harder than actual pulmonary tumors. Once the pseudo PPLs were ready, their locations were indicated by the blue color of the indigocarmine and visualized from the lung surface. Finally, the radial probe was re-inserted in the bronchi in order to confirm that clear ultrasound images of the target lesions could be obtained.

\section{Equipment for EBUS-GS procedures}

All EBUS-GS procedures were performed using BF-1T260 (5.9-mm outer diameter, 2.8-mm working channel diameter; Olympus, Japan) in combination with a radial probe (UMS20-20R; Olympus, Japan) and a large GS kit (K-203; Olympus, Japan) including biopsy forceps. Bronchoscopy was performed through the oral route of the ArtiCHEST ${ }^{\mathrm{TM}}$ system.

\section{Hands-on training program}

A total of 12 voluntary trainees participated in a hands-on training course for EBUS-GS using our biosimulator. The experience level as a bronchoscopist was not considered as a criterion for participation in this course. The trainees were informed in advance that they would be using a new training model based on ex vivo porcine lungs with pseudo PPLs. However, the detail content of this study was not informed to the trainees.

The goal of the hands-on course was to enable the participants to perform the EBUS-GS procedure precisely and acquire adequate samples from target lesions. The first half of the program included a 30-min lecture about EBUSGS, while the latter half involved hands-on training using the biosimulator. At the beginning of the hands-on training course, trainers demonstrated and explained the following: (I) how to set up the EBUS-GS device; (II) how to approach the target lesion; (III) how to acquire appropriate EBUS images of the target lesion as hypoechoic areas, and (IV) how to acquire samples using biopsy forceps. The trainees were divided into three groups ( $\mathrm{n}=4$ each), with one group undergoing training using a single simulator for 40 minutes. In each group, two trainees together performed EBUSGS on the biosimulator and obtained a sample from a pseudo PPL; this was considered the initial biopsy. Then, the trainers gave feedback advice to the trainees, focusing on techniques for acquiring larger samples. In particular, they advised the trainees to close the forceps cups slowly while pushing the forceps by several millimeters through GS against the target lesion. Subsequently, the trainees performed 2 -5 procedures as training, and they again acquired samples from the same target lesion; this was considered the post-training biopsy. The acquired samples were stocked in a plastic container in a wet environment, and the sample size was compared between the initial and post-training biopsies.

After the training program, all trainees answered a questionnaire regarding the usefulness of the biosimulator and their satisfaction with the training program.

\section{Results}

The characteristics of the 12 trainees with regard to the duration of clinical practice and experience with EBUSGS are shown in Table 1. Under the trainer's guidance, all trainees successfully detected pseudo PPLs and obtained clear images of hypoechoic areas using radial probe EBUS 
before the initial biopsy, and 11 of them acquired blue samples in the initial biopsy. One trainee only acquired blue solution without a solid specimen. In the post-training biopsy, eight trainees acquired samples larger than the initial samples (Figure 3). One trainee acquired a smaller sample in the post-training biopsy. Pneumothorax in the porcine lungs did not occur after any procedure.

The results of the questionnaire revealed that all trainees were satisfied with the biosimulator. Moreover, eight trainees who had previously participated in another handson training program based on a synthetic phantom model were more satisfied with the biosimulator than with the synthetic phantom model (Table 2).

\section{Discussion}

This study demonstrated the usefulness of a novel

Table 1 Characteristics of the trainees selected for hands-on training with a novel biosimulator for peripheral pulmonary lesions sampling using EBUS-GS

\begin{tabular}{ll}
\hline Characteristics & $\mathrm{N}=12$ \\
\hline Duration of clinical practice (years) & \\
$<4 / 4-10 />10$ & $7 / 4 / 1$
\end{tabular}

Experience in EBUS-GS (years)

$$
0 /<1 / 1-3 />3
$$

Number of EBUS-GS procedures performed

$$
\text { 0/1-10/11-50/>51 }
$$

EBUS-GS, endobronchial ultrasonography with a guide sheath. biosimulator based on ex vivo porcine lungs with pseudo PPLs for training in peripheral tissue sampling using EBUS-GS. The trainees could successfully acquire samples from the target lesions, with some post-training samples being larger than those obtained before training. In addition, clear sonographic images of PPLs, similar to those of actual PPLs in human lungs, were obtained. The results of a questionnaire administered to the trainees revealed that the biosimulator and the hands-on training program were useful and helped the trainees in acquiring the required

Table 2 Results of a questionnaire for assessing trainee satisfaction levels with regard to a novel biosimulator created for hands-on training in peripheral pulmonary lesions sampling using EBUS-GS

\begin{tabular}{lc}
\hline Questions & Yes \\
\hline $\begin{array}{l}\text { The biosimulator helped in understanding the } \\
\text { EBUS-GS procedure }\end{array}$ & $11(92 \%)$
\end{tabular}

The biosimulator helped in the detection of target

$12(100 \%)$ peripheral pulmonary lesions using radial EBUS

The biosimulator helped in improving skills for $11(92 \%)$ acquiring samples with biopsy forceps

The biosimulator was better than a synthetic phantom model for training in EBUS-GS*

You were satisfied with the hands-on training using $12(100 \%)$ the biosimulator

Each question had three multiple choices as answers: yes, no, and N/A. *, this question was given to eight trainees who had experienced hands-on training with a synthetic phantom model. EBUS-GS, endobronchial ultrasonography with a guide sheath; N/A, not applicable.
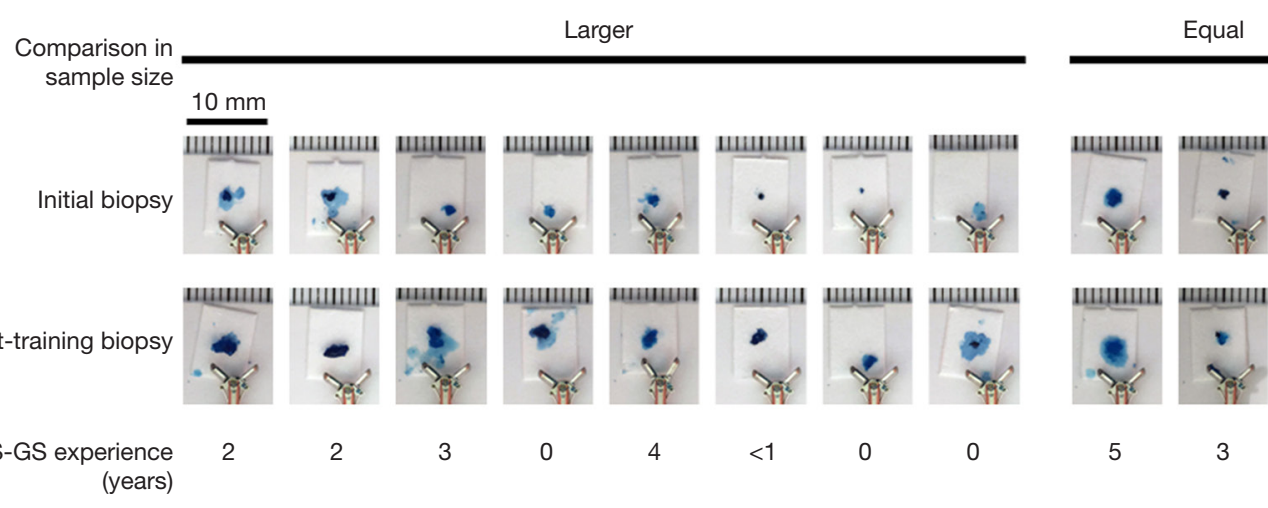

Smaller

Figure 3 Comparison of sample sizes between initial and post-training biopsies performed using EBUS-GS. A novel biosimulator with pseudo peripheral pulmonary lesions was used to train the participants in peripheral tissue sampling using EBUS-GS. It can be seen that the post-training samples are larger than the initial samples for eight of the 12 trainees. One post-training sample is smaller than the initial sample. EBUS-GS, endobronchial ultrasonography with a guide sheath. 
knowledge and skills for EBUS-GS.

Previous reports on the clinical education system suggested that simulation-based training encourages trainees to learn from errors in an error-forgiving environment $(10,11)$ where there are no serious complications caused by lack of experience. Moreover, simulators, including synthetic phantoms, cadaveric models, and models based on virtual reality, initially enhance the learning curve for beginners (12). Among these simulators, training models based on virtual reality, also known as high-fidelity simulators, allow operators to navigate through computer-generated airway images. Virtual reality helps trainees in improving their recognition of pertinent anatomical structures and acquiring basic dexterity and skills (13). However, a prospective, randomized crossover study by Davoudi et al. revealed that a low-fidelity model made from molded silicone or excised animal airways was superior to a high-fidelity simulator for training in conventional transbronchial needle aspiration (TBNA) (14). The authors discussed that a low-fidelity model was more suitable and advantageous for learning detailed maneuvers because it is more real and allows for practice using actual instruments. In recent studies, a new three-dimensional (3D)-printed simulator was reported to be useful as a low-fidelity model for training in bronchoscopy $(15,16)$. The advantages of this $3 \mathrm{D}$-printed simulator include the low cost and possibility of anatomical correction because of the use of computed tomography (CT) data obtained from an actual patient. However, because the model is made from plastic filaments, it is not very real in terms of mechanical properties such as hardness and skid resistance on the bronchial surface. In contrast, porcine lungs, which were used in the present study, closely approximate the tissue characteristics of the human lungs, although there is a slight difference in the anatomy.

The pulmonary biosimulator established in the present study was originally inspired by another training simulator for EBUS-guided TBNA (EBUS-TBNA) (17). Because our novel biosimulator comprised porcine lungs with pseudo PPLs, the trainees could learn how to select the target bronchi and insert the bronchoscope and GS under actual clinical conditions involving human lungs with PPLs. In the present study, we transbronchially injected colored agar solution through GS into the peripheral lung field of the porcine lungs. This transbronchial injection through GS resulted in the creation of pseudo PPLs with a bronchus sign (18), which is one of the most important predictive factors for obtaining correct samples and diagnosing PPLs (19-21). If the agar is injected from outside the lungs, formation of pseudo PPLs with the bronchus sign and confirmation of the target bronchi for EBUS-GS may take longer. We did not use a transbronchial needle to inject agar into the peripheral lung fields because pseudo PPLs for hands-on training in transbronchial forceps biopsy should be inside the bronchi; in addition, the porcine lungs should be free of pneumothorax for training purposes. Transbronchial injection of agar through GS was quick and easy, and the addition of indigocarmine to the agar made it easy to identify the PPLs locations from outside the lungs and confirm whether the acquired samples contained the target tissue. This helped the trainees in understanding whether or not their procedures were successful.

The training program using our biosimulator helped trainees in acquiring larger samples using biopsy forceps inserted through GS. Bronchoscopists are expected to acquire an adequate sample amount for not only diagnosis but also gene analysis, because gene mutation analysis is essential for deciding the treatment for non-small cell lung cancer (22). In our hands-on training program, trainers advised the participants on the efficient usage of biopsy forceps, teaching them to slowly close the forceps cups while pushing the forceps with GS against the target by several millimeters. This technique, which enables the collection of large tissue samples in the forceps cups, has been explained as a tip for improving the adequacy of specimens obtained by biopsy forceps in some textbooks or papers on gastrointestinal endoscopy $(23,24)$ and is also used by some bronchoscopists in clinical practice.

The present study has several limitations. First, it included a small number of participants. Although the hands-on training program using the biosimulator increased the understanding of trainees and helped them in acquiring larger samples, further research with a larger number of participants is necessary to confirm the effectiveness of this model. Moreover, we investigated the utility of this model by involving trainees with various levels of experience in bronchoscopy. A previous research about a benefit of EBUSTBNA training using virtual reality simulator demonstrated that novices acquired significant improvement in their skills, while experts did not benefit from the simulation training (25). Future studies should also investigate the experience level at which this training model is most effective. Second, we did not conduct an objective evaluation of the biosimulator model using quantitative scoring assessment and comparing with other training models such as synthetic phantoms. Third, the time for hands-on training was not long enough. The trainees performed several procedures in this training 
program. Although there are little evidence that show the number of procedures required for competent radial probe EBUS performance, it is said that some dozens of procedures on simulators are needed (26). A training program with enough long time for performing many procedures will be needed to assess the new training model correctly. Fourth, although our hands-on training program succeeded in improving the sample size, the quality of specimen, such as the proportion of normal lung tissue and crash artifacts, could not be assessed.

\section{Conclusions}

We established and evaluated the feasibility of a novel biosimulator based on porcine lungs with pseudo PPLs for training in peripheral tissue sampling using EBUS-GS. A hands-on training program using this biosimulator could help trainees in understanding and acquiring the skills for EBUS-GS and learning techniques for obtaining larger samples through biopsy forceps. A structured training program with more procedures and a specific direct observation of procedural skills is needed to confirm the validity of the new biosimulator.

\section{Acknowledgments}

None.

\section{Footnote}

Conflicts of Interest: T Ishiwata and T Nakajima received honoraria and lecture fees from AstraZeneca and Olympus Medical Systems for training courses. The training program in the present study was supported by AstraZeneca and Olympus Medical Systems. These sponsors had no role in the study design, conduct of the study, data collection, data management and interpretation, preparation of the report or approval of the report.

Ethical Statement: The authors are accountable for all aspects of the work in ensuring that questions related to the accuracy or integrity of any part of the work are appropriately investigated and resolved.

\section{References}

1. Herth FJ, Ernst A, Becker HD. Endobronchial ultrasound-guided transbronchial lung biopsy in solitary pulmonary nodules and peripheral lesions. Eur Respir J 2002;20:972-4.

2. Kurimoto N, Miyazawa T, Okimasa S, et al. Endobronchial ultrasonography using a guide sheath increases the ability to diagnose peripheral pulmonary lesions endoscopically. Chest 2004;126:959-65.

3. Bolliger CT, Mathur PN, Beamis JF, et al. ERS/ATS statement on interventional pulmonology. European Respiratory Society/American Thoracic Society. Eur Respir J 2002;19:356-73.

4. Ernst A, Silvestri GA, Johnstone D. Interventional pulmonary procedures: Guidelines from the American College of Chest Physicians. Chest 2003;123:1693-717.

5. Du Rand IA, Barber PV, Goldring J, et al. British Thoracic Society guideline for advanced diagnostic and therapeutic flexible bronchoscopy in adults. Thorax 2011;66 Suppl 3:iii1-21.

6. Ernst A, Wahidi MM, Read CA, et al. Adult Bronchoscopy Training: Current State and Suggestions for the Future: CHEST Expert Panel Report. Chest 2015;148:321-32.

7. Eom JS, Mok JH, Kim I, et al. Radial probe endobronchial ultrasound using a guide sheath for peripheral lung lesions in beginners. BMC Pulm Med 2018;18:137.

8. Uchimura K, Yamasaki K, Ishimoto H, et al. Factors Associated with Diagnostic Yield of Endobronchial Ultrasonography with a Guide Sheath for Peripheral Lung Cancer. J uoeh 2016;38:17-23.

9. Ishiwata T, Nakajima T, Terada J, et al. Preparation of a novel biosimulator with pseudo peripheral pulmonary lesions for training in peripheral tissue sampling using endobronchial ultrasonography (EBUS) with a guide sheath (EBUS-GS). Asvide 2019;6:312. Available online: http://www.asvide.com/watch/32997

10. Ziv A, Ben-David S, Ziv M. Simulation based medical education: an opportunity to learn from errors. Med Teach 2005;27:193-9.

11. Cook DA, Hatala R, Brydges R, et al. Technologyenhanced simulation for health professions education: a systematic review and meta-analysis. JAMA 2011;306:978-88.

12. Ziv A, Wolpe PR, Small SD, et al. Simulation-based medical education: an ethical imperative. Acad Med 2003;78:783-8.

13. Colt HG, Crawford SW, Galbraith O 3rd. Virtual reality bronchoscopy simulation: a revolution in procedural training. Chest 2001;120:1333-9.

14. Davoudi M, Wahidi MM, Zamanian Rohani N, et al. Comparative effectiveness of low- and high-fidelity 
bronchoscopy simulation for training in conventional transbronchial needle aspiration and user preferences. Respiration 2010;80:327-34.

15. Pedersen TH, Gysin J, Wegmann A, et al. A randomised, controlled trial evaluating a low cost, 3D-printed bronchoscopy simulator. Anaesthesia 2017;72:1005-9.

16. Parotto M, Jiansen JQ, AboTaiban A, et al. Evaluation of a low-cost, 3D-printed model for bronchoscopy training. Anaesthesiol Intensive Ther 2017;49:189-97.

17. Nakajima T, Fujiwara T, Saegusa F, et al. Specimen acquisition training with a new biosimulator in endobronchial ultrasound-guided transbronchial needle aspiration. Medicine (Baltimore) 2017;96:e6513.

18. Tsuboi E, Ikeda S, Tajima M, et al. Transbronchial biopsy smear for diagnosis of peripheral pulmonary carcinomas. Cancer 1967;20:687-98.

19. Evison M, Crosbie PA, Morris J, et al. Can computed tomography characteristics predict outcomes in patients undergoing radial endobronchial ultrasound-guided biopsy of peripheral lung lesions? J Thorac Oncol 2014;9:1393-7.

20. Guvenc C, Yserbyt J, Testelmans D, et al. Computed tomography characteristics predictive for radial EBUSminiprobe-guided diagnosis of pulmonary lesions. J

Cite this article as: Ishiwata T, Nakajima T, Terada J, Tatsumi $\mathrm{K}$. A novel biosimulator based on ex vivo porcine lungs for training in peripheral tissue sampling using endobronchial ultrasonography with a guide sheath. J Thorac Dis 2019;11(10):4152-4158. doi: 10.21037/jtd.2019.10.05
Thorac Oncol 2015;10:472-8.

21. Ali MS, Trick W, Mba BI, et al. Radial endobronchial ultrasound for the diagnosis of peripheral pulmonary lesions: A systematic review and meta-analysis. Respirology 2017;22:443-53.

22. Kim L, Tsao MS. Tumour tissue sampling for lung cancer management in the era of personalised therapy: what is good enough for molecular testing? Eur Respir J 2014;44:1011-22.

23. Levine DS, Reid BJ. Endoscopic biopsy technique for acquiring larger mucosal samples. Gastrointest Endosc 1991;37:332-7.

24. Gershman G, Thomson M. Practical Pediatric Gastrointestinal Endoscopy. 2nd edition. Wiley-Blackwell, 2012.

25. Scarlata S, Palermo P, Candoli P, et al. EBUS-STAT Subscore Analysis to Predict the Efficacy and Assess the Validity of Virtual Reality Simulation for EBUSTBNA Training Among Experienced Bronchoscopists. J Bronchology Interv Pulmonol 2017;24:110-6.

26. Livi V, Barisione E, Zuccatosta L, et al. Competence in navigation and guided transbronchial biopsy for peripheral pulmonary lesions. Panminerva Med 2019;61:280-9. 